\title{
Geometric Correction and Validation of Hyperion and ALI Data for EVEOSD
}

\author{
Andrew Dyk ${ }^{1}$, David G. Goodenough ${ }^{1,2}$, A. S. (Pal) Bhogal ${ }^{1}$, Jay Pearlman ${ }^{3}$, and Justin Love ${ }^{2}$ \\ ${ }^{1}$ Pacific Forestry Centre, Natural Resources Canada \\ ${ }^{2}$ Department of Computer Science, University of Victoria \\ ${ }^{3}$ TRW Inc. \\ 506 West Burnside Road, Victoria, BC, Canada, V8Z 1M5 \\ (250-363-0153, office; 250-363-0775, fax); adyk@nrcan.gc.ca
}

\begin{abstract}
Precise geometric correction of EO-1's Hyperion data is essential to link ground spectral data and satellite hyperspectral data. Two scenes have been selected from sites of the EVEOSD (Evaluation and Validation of EO-1 for Sustainable Development of Forests) project. One site is the Greater Victoria Watershed District (GVWD) located on south Vancouver Island, $\mathrm{BC}$ and the other is Hoquiam located in southwestern Washington State. Ground Control Point (GCP) collection has been performed using a feature fitting method in which high accuracy, orthorectified photo-derived polygons of features are used for tie-down. For example lakes are adjusted to match the same feature obvious in the hyperspectral imagery. This technique allows for easier estimation of a GCP's precise fit to the imagery. A third (11) of the GCPs were identified as check points to validate the geometric models. GCPs were collected independently from both the VNIR and SWIR arrays of the Hyperion sensor to determine the adjustment factor required to remove the displacement and skew between these arrays. The adjustment can then be applied to GCPs collected from one array to make a compatible geometric correction model for both arrays. The polynomial and rational function correction methods have been applied to both scenes with various orders applied to each function. The effect of terrain distortion removal is evaluated in using the rational function method.

Hyperion data can be geocorrected with surprising accuracy. For example, we obtained $10 \mathrm{~m}$ RMS on check points with the rational function. With a second order polynomial we achieved $13 \mathrm{~m}$ RMS without terrain correction. The accuracy of this latter result is due to the small swath width of the sensor. Applying terrain correction does improve the accuracy of geometric correction in areas with high relief. A similar procedure was applied to EO-1's ALI sensor and this paper compares the results for Hyperion and ALI geometric fidelity.
\end{abstract}

\section{INTRODUCTION}

Precise geometric correction of remotely sensed data is critical if ground data is used for sensor validation. The project Evaluation and Validation of EO-1 for Sustainable Development of forests (EVEOSD) [1], in cooperation with NASA, evaluates the ability of multiple sensor to measure forest attributes. Relating foliar sample, land cover GIS data and spectral ground measurements to the data acquired from multiple sensors requires a good geometric correction of imagery.

The EVEOSD project is also validating EO-1 Hyperion imagery by comparing it with AVIRIS hyperspectral data and ALI to ETM+ multispectral. This paper discusses the process of correcting EO-1 Hyperion and ALI data.
Orthorectification of Landsat's ETM+ data has been performed using rigorous sensor models as implemented in PCI software. The RMS for the ETM+ multispectral image's 21 GCPs was $8.24 \mathrm{~m}$, and $7.80 \mathrm{~m}$ for the 8 check GCPs.

The first EVEOSD test-sites that required geometric correction of EO-1 data were the Greater Victoria Watershed District (GVWD) located on south Vancouver Island, BC and Hoquiam, located in southwestern Washington State. The GVWD test site contains some of the oldest stands of Douglas Fir in the southern half of Vancouver Island. The average elevation of the GVWD is about 400 meters above sea level, with slopes as great as 45 degrees for some of the plots. Except for the younger stands, the old growth forest in this test site is largely unmanaged, and most of our experimental plots are from unmanaged areas.

The Hoquiam test site contains Weyerhaeuser research plots for fertilization study of Douglas Fir and a mixed forest research site of Douglas Fir and Western Hemlock. The mean elevation for the Hoquiam study site is 100 meters above sea level.

NASA's EO-1 satellite successfully acquired imagery over the Hoquiam test site on August 9, 2001 and over the GVWD on September 10, 2001.

The next section of this paper describes a GCP collection method that is independent of the correction model and uses a thin-plate spline (TPS) to inversely rubber sheet vectorbased features over the uncorrected imagery, to determine GCPs. The GCPs collected independently from Hyperion's VNIR and SWIR spectrometers determine the registration correction that can then be applied to GCPs collected from each spectrometer. The effects of geometric correction on the spectra are analyzed.

ALI data were corrected by collecting GCPs on the panchromatic band, then a relationship was developed to adjust the GCPs for each ALI multispectral band, to account for the band to band misregistration. Orthorectifying the imagery was performed using the PCI Rational Function Model (RFM) based on the GCPs derived for each Hyperion array and the GCPs derived for each band of ALI. Comparisons are made between the use of RFM and a polynomial correction. 


\section{GCP COLLECTION}

Collection of accurate GCPs is the basis for good geometric correction. In the GVWD study area clear road intersection are not abundant throughout. There are, however, numerous large and small lakes and reservoirs. Accurate GCPs are established from polygon vectors such as lakes by inversely rubber sheeting the vectors to fit the imagery. This establishes the relationship of a mapping coordinate, from somewhere in the middle of the lake, to a fractional pixelline coordinate in the imagery. For example, the edge of the lake vector is aligned with the shoreline surrounding the lake in the imagery. If the lake in the imagery appears as a dark feature, surrounded by brighter land pixels, the shoreline pixels are a shade of gray. The lake edges of the vector are moved to fit such that they cover only the gray pixels. Fig. 1 shows how a vector edge looks slightly misregistered and then properly registered. The adjustment between these two images was only 0.3 of a pixel in both the $\mathrm{x}$ and $\mathrm{y}$ directions.

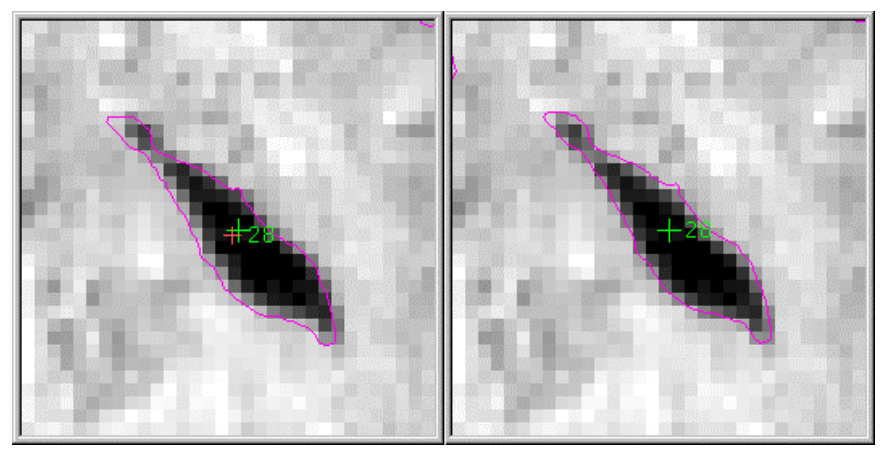

Fig. 1. The effect of using TPS to shift a GCP 0.3 pixels right and 0.3 pixels up to best fit the feature.

The requirement of the source vector is that they are of an accuracy greater than the size of the pixels being corrected. For the GVWD the vectorized lakes are from the CRD Watershed district, and their accuracy is $\sim 10 \mathrm{~m}$. For Hoquiam, differentially corrected GPS data were used.

Since the lakes represent topographically flat areas, there is no variable distortion in the imagery caused by topographic effects. The intersection of two streams flowing down a hill can be aligned with TPS to fit on the imagery, but the river vectors will not overlay on the imagery properly as the distance from that intersection increases. This is due to the topographic distortions in the imagery that are not being accounted for in the rubber sheeting model of TPS. The GCPs at the river intersection would still be valid but it is difficult to confirm the fit of the river feature the further one looks away from that intersection.

The GCPs then have an elevation attached to them by extracting the heights from a DEM. The GCPs can be used to drive any geometric correction algorithm such as polynomial, RFM or a rigorous model.

\section{HyPERION REgISTRATION}

The VNIR and SWIR spectrometers are two different instruments which share the same fore-optics[2]. These arrays can not be perfectly overlaid before geometric correction as there is a sub-pixel misregistration that varies across track. To determine this distortion, a relationship was determined between the pixel locations of GCPs collected from the two arrays. The pixel-line coordinates of GCPs collected from one array were visually adjusted to fit the other, and the pixel-line adjustment factor was determined. This factor can then be applied to the GCPs for any Hyperion image collected from a single array. The resulting geometrically corrected image with the two arrays is now properly registered.

For the Hyperion image over the GVWD, 40 GCPs where collected over a $30 \mathrm{~km}$ long strip. Lake vectors were fitted over the SWIR image using the TPS method as described in the previous section. The pixel-line coordinates of these GCPs where then adjusted to fit over the VNIR image, resulting in each set of GCPs having the same map coordinates. A first order polynomial regression was developed to fit the SWIR pixel-line coordinates to the VNIR pixel-line coordinates. Table 1 shows the coefficients of this polynomial fit, which has a RMS of 0.23 pixels.

TABLE 1

SWIR/VNIR PIXEL/LINE REGRESSION COEFFICIENTS

\begin{tabular}{|r|r|c|}
\hline \multicolumn{2}{|c|}{ Forward (SWIR-VNIR) } & Backward (VNIR-SWIR) \\
\hline Fx_Const & -0.6352552 & 0.6359172 Bx_Const \\
Fx_X & 0.9985208 & 1.0014770 Bx_X \\
Fx_Y & -0.0000308 & -0.0000310 Bx_Y \\
\hline Fy_Const & -0.2085051 & 0.2067326 By_Const \\
Fy_X & 0.0036988 & -0.0037045 By_X \\
Fy_Y & 1.0000440 & $0.9999557 \mathbf{B y} \mathbf{B}$ \\
\hline
\end{tabular}

Fitting the SWIR array to the VNIR, the adjustment in the X direction can be simplified if $\mathbf{F x} \mathbf{X}$ is rounded to 1 and Fx_Y to 0 , resulting in a constant minus two thirds (Fx_const) of a pixel as expressed in (1). Equation (2) shows a shift in the $\mathrm{Y}$ direction as a function of its $\mathrm{X}$ position (a skew). The arrays line up on the west side and increase to a discrepancy of around one pixel (0.95) on the east side. Equations (3) and (4) show the relationship of VNIR to SWIR array.

$$
\begin{aligned}
& S W I R x=\text { VNIRx }+0.635 \\
& \text { SWIRy }=\text { VNIRy }-(\text { VNIRx } / 270)+0.032 \\
& V N I R x=\text { SWIRx }-0.635 \\
& V N I R y=\text { SWIRy }-(\text { SWIRx / 270) }-0.034
\end{aligned}
$$

\section{ALI Registration}

The ALI sensor has nine $30 \mathrm{~m}$ multispectral bands and 1 $10 \mathrm{~m}$ panchromatic channel. A misregistration of the multispectral bands requires GCPs be collected separately for each channel. A relationship between each channel's mis- 
registration can be established to a base line. The fourth Sensor Chip Assembly [3] (M4) in the ALI sensor was chosen for geometric correction as it overlaps with the Hyperion footprint. The $10 \mathrm{~m}$ panchromatic channel was chosen to be the baseline image for GCP collection since the higher spatial resolution allows for the collection of more accurate GCPs. The relationship between the same GCPs collected from the panchromatic channel to each of the multispectral channels was determined by shifting the pixel-lines of GCPs collected from the panchromatic band to match each multispectral band. Some features were very difficult to see on some bands, so these points where not used. Of the original 40 GCPs collected from the panchromatic, 28 could be identified in all bands. The average pixel and line difference for each band can be seen in Fig. 2. There is a trend that as the band number increases, the pixels shift by over a third of a pixel down, and a quarter of a pixel right.

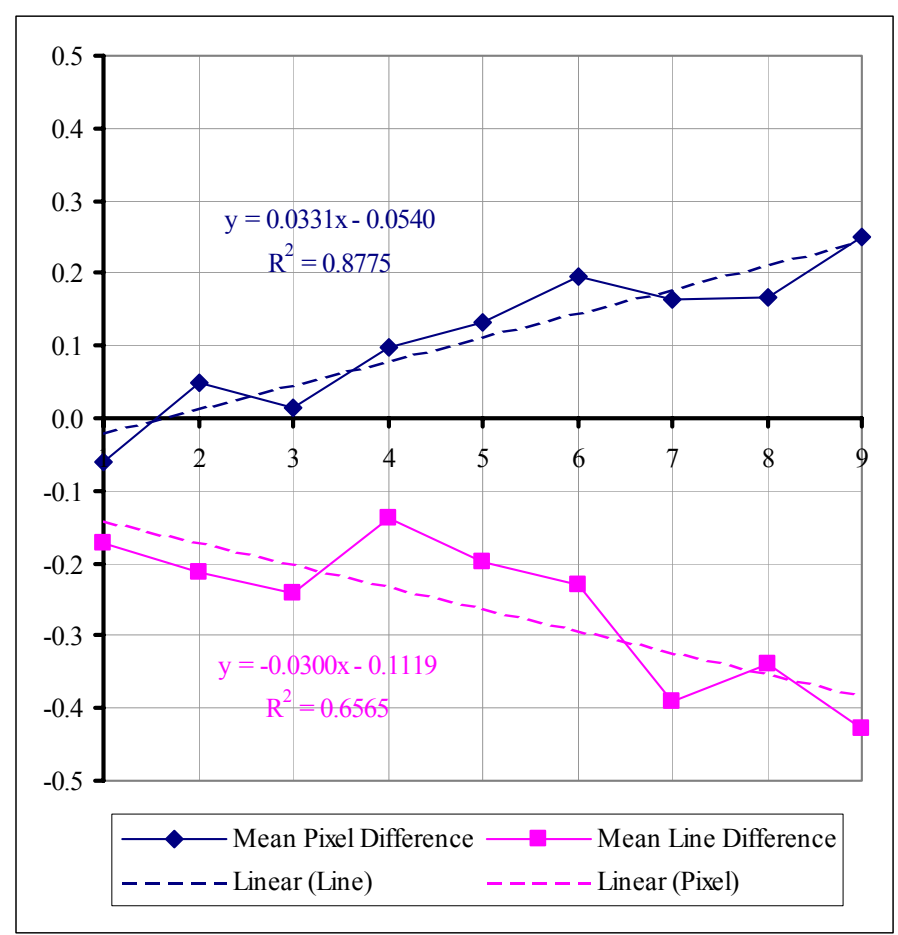

Fig. 2. Average difference of pixel and line coordinates of normalized panchromatic GCPs and each ALI multispectral band.

ALI's M4 panchromatic imagery is buffered in the top. The number of lines in this buffer was determined by resampling ALI band 2 to 10 meters, then overlaying this band on the panchromatic data. The position of the resampled band 2 was adjusted until a visual best fit was made. The result of this exercise revealed there to be 513 lines in the buffer. There does not appear to be any shift across track.

Equations (5) and (6) convert the pixel-line coordinates of the panchromatic channels of ALI's M4 array to the equivalent pixel-line coordinate of the multispectral channels where MSpixel is the multispectral GCP pixel position, Ppixel is the panchromatic GCP pixel, MSline is the multis- pectral GCP line, Pline is the panchromatic GCP line, and Npixel is the multispectral GCP pixel for channel $\mathrm{N}$ and Nline is the multispectral GCP for channel N.

$$
\begin{aligned}
& \text { MSpixel }=\text { Ppixel } / 3-\text { Npixel } \\
& \text { MSline }=\text { Pline / } 3-513-\text { Nline }
\end{aligned}
$$

The one-third factor adjusts for the $10 \mathrm{~m}$ to $30 \mathrm{~m}$ pixel sizes of the panchromatic and multi-spectral sensors. Table 2 shows the channel dependant constants Nline and Npixel. These adjustment factors show the amount of shift between each band.

TABLE 2

CONSTANTS TO BE ADDED TO THE ALI M4 PANCHROMATIC CHANNEL DURING CONVERSION TO EQUIVALENT MULTISPECTRAL COORDINATES

$\begin{array}{cccr}\text { Channel } & \text { Band } & \text { Ave_Pix_Dif Ave_Lin_Dif } \\ 1 & (1 \mathrm{p}) & -0.06026 & -0.17315 \\ 2 & (1) & 0.04742 & -0.21262 \\ 3 & (2) & 0.01499 & -0.24198 \\ 4 & (3) & 0.09643 & -0.13738 \\ 5 & (4) & 0.13302 & -0.19826 \\ 6 & (4 \mathrm{p}) & 0.19474 & -0.23058 \\ 7 & (5 \mathrm{p}) & 0.16360 & -0.39190 \\ 8 & (5) & 0.16642 & -0.34044 \\ 9 & (7) & 0.24881 & -0.42833\end{array}$

V. ORthorectification

Geometrically correcting the images required taking the GCPs collected and applying them to a geometric correction algorithm. The EO-1 satellite's physical sensor model is not well enough known to apply a rigorous model orthorectification. The rational function model (RFM) is a generalized sensor model that uses the ratios of polynomials to establish the relationship between the image coordinates and the object coordinates [4]. The model parameters are derived from the GCPs themselves and a $25 \mathrm{~m}$ gridded DEM as implemented in the PCI Ortho-engine software.

\section{A. GVWD Hyperion}

Forty GCPs were collected for the Hyperion image over the GVWD. Eleven were used as check points and 29 were used for geometric correction models. Table 3 shows the total RMS of the GCPS and check points in meters when using a polynomial correction with $1^{\text {st }}$ and $2^{\text {nd }}$ order and the RFM with 3 to 11 coefficients.

The polynomial corrections produced a RMS of only half a pixel. The good results are due in part to the small swath width of the Hyperion sensor. The error in the $\mathrm{X}$ direction was greater than the $\mathrm{Y}$ direction, illustrating the distortion across track caused by terrain displacement in the GVWD.

RFM errors drop immediately and level off to one-third of a pixel as the number of coefficients increases over three. The SWIR array bands were corrected with the same GCPs 
on the VNIR, after they were adjusted for the array shifts. The result is that both arrays have the same RMS errors.

TABLE 3

GVWD HYPERION RMS ERRORS IN METERS

\begin{tabular}{|c|c|c|c|c|c|c|}
\hline & \multicolumn{3}{|c|}{ GCP $n=29$} & \multicolumn{3}{|c|}{ Check Point n=11 } \\
\hline & GCP-X & GCP-Y & RMS & Check-X & Check-Y & RMS \\
\hline \multicolumn{7}{|c|}{ Polynomial } \\
\hline 1 & 14.71 & 6.15 & 15.94 & 16.23 & 8.34 & 18.25 \\
\hline 2 & 7.84 & 4.69 & 9.14 & 10.32 & 7.85 & 12.97 \\
\hline \multicolumn{7}{|c|}{ Rational Function } \\
\hline 3 & 13.04 & 5.67 & 14.22 & 16.17 & 8.13 & 18.10 \\
\hline 4 & 4.36 & 4.50 & 6.27 & 7.53 & 6.89 & 10.21 \\
\hline 5 & 4.02 & 4.44 & 5.99 & 7.36 & 6.85 & 10.05 \\
\hline 6 & 4.04 & 4.19 & 5.82 & 7.37 & 6.89 & 10.09 \\
\hline 7 & 4.01 & 4.18 & 5.79 & 7.52 & 6.82 & 10.15 \\
\hline 8 & 3.98 & 3.98 & 5.63 & 7.71 & 7.06 & 10.45 \\
\hline 9 & 3.94 & 4.02 & 5.63 & 7.42 & 7.06 & 10.24 \\
\hline 10 & 3.62 & 2.82 & 4.59 & 7.41 & 6.33 & 9.75 \\
\hline 11 & 3.49 & 2.79 & 4.47 & 7.29 & 6.27 & 9.62 \\
\hline
\end{tabular}

Six coefficients in the RFM were selected with an output pixel size of $25 \mathrm{~m}$. The resampling kernel used was PCI's implementation of a 16 point $(\sin (\mathrm{X}) / \mathrm{X})^{2}$ function.

Resampling of hyperspectral imagery has an effect on the spectra. Unfortunately PCI's implementation of the 16 point $(\sin (\mathrm{X}) / \mathrm{X})^{2}$ function is not truncated correctly for 12-bit data, and spurious negative values appear in the geometrically corrected image. All of these negative values have been set to zero in the geometrically corrected image. The comparison of three types of spectra collected from both the original uncorrected imagery and corrected imagery can be found in Fig. 2. Regions of interest (ROI) of $3 \times 3$ pixels were identified over the $30 \mathrm{~m}$ uncorrected imagery and the equivalent $4 \times 4$ pixels ROI were visually positioned over the $25 \mathrm{~m}$ geocorrected data. The three spectra sampled were herb graminoid from a farmer's field, a mature Douglas Fir stand and water from Shawnigan Lake. Fig. 3 shows the difference in raw DN of the uncorrected spectra minus the geocorrected spectra.

Collecting spectra from the uncorrected data required adjusting the ROIs for the VNIR and SWIR to account for the co-alignment. The greatest variation in the spectra is a difference of $-182 \mathrm{DN}$ found in the Herb Graminoid spectra at $1034 \mathrm{~nm}$. The original spectra mean for this ROI is a DN of 4972, so the difference is $3.66 \%$. This difference may be due a misregistration of the corrected and uncorrected ROIs. The average absolute DN difference is 20.2 for Herb Graminoid, 7.1 for Douglas Fir and 5.6 for water.

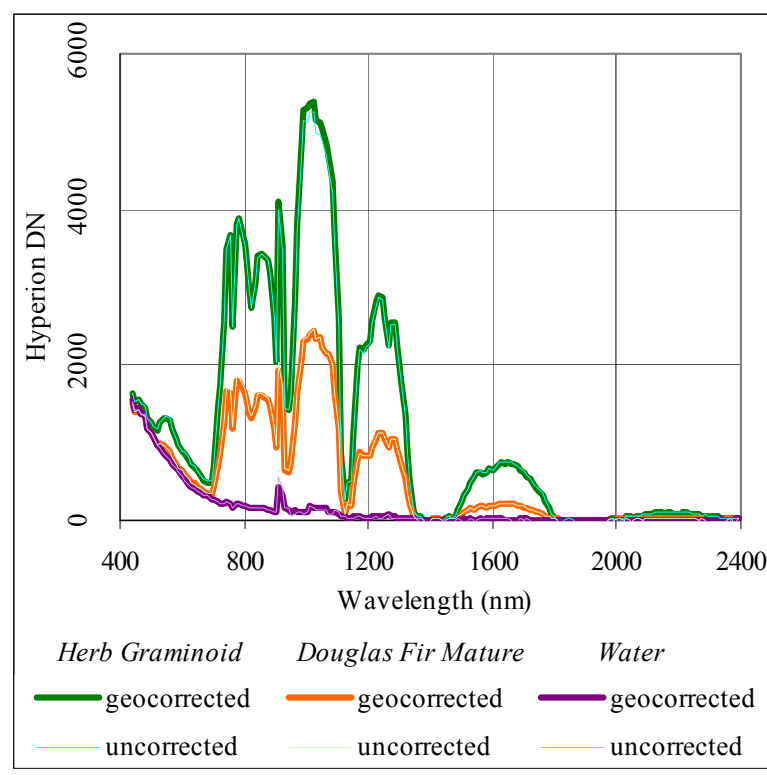

Fig. 2. Mean RAW DN spectral comparisons before and after geocorrection for three classes.

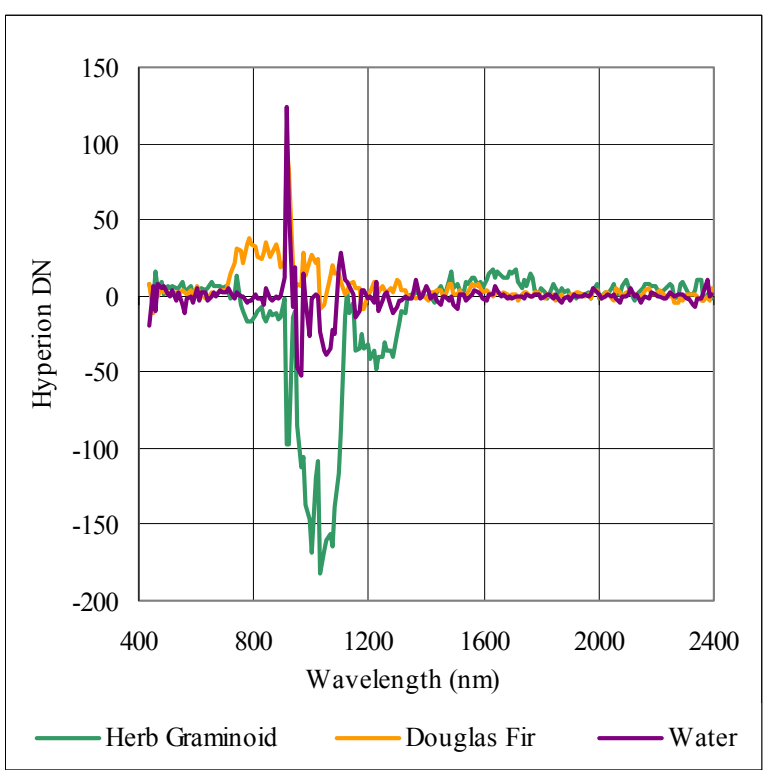

Fig. 3. Spectral differences before and after geocorrection.

\section{B. Hoquiam Hyperion}

The Hyperion image for Hoquiam received from TRW Inc. was custom processed to a Level L1PS2. The letter "P" meant that the VNIR/SWIR co-alignment has been performed before geometric correction. The SWIR array was moved one pixel to the left, and all pixels on the right hand side of the array were shifted down one line, resulting in a file that is only 255 pixels wide, one less than data that hasn't had the co-alignment performed to it. This coalignment method is a non-resampling approximation of the array, and spectra collected from the non-geocorrected data can be viewed. There is an obvious visual discontinuity in 
the SWIR array where the middle pixels have been shifted down one line.

The co-alignment had to be undone in order to apply the GCP method of co-alignment. The image was copied into a file 256 pixels wide and the VNIR channels were left justified. The SWIR channels were copied into the new file, one half at a time. The left side (pixels 1-126) was placed one pixel from the left edge of the new file (pixels 2 to 127). The right half of the array (pixels 127 to 255) was placed into the new file one pixel to the left (pixels 128-256) and one pixel up to their original position.

Forty GCPs were collected from the VNIR channel by fitting them to differentially corrected GPS data collected while driving in the area, as well as 1 meter ortho-photos supplied by Weyerhaeuser. The forest cover data were available, but it was not created from ortho-rectified aerial photos. The forest cover data were not used in the geometric correction process. One third of the GCPs were held back for checkpoints to validate the RMS of the correction methods. The RMS of the 29 GCPs was $5.82 \mathrm{~m}$ and for the checkpoints was $10.09 \mathrm{~m}$ when using a rational function with six coefficients. The SWIR bands were adjusted using the ' -0.25 to 1 and -1 ' method as suggested by [5]. SWIR GCPs were shifted to match the VNIR by -0.25 lines at pixel 1 and by 1 line at pixel 256. A constant one pixel shift was applied in the along track direction.

\section{GVWD ALI}

The ALI image acquired over the GVWD was corrected using GCPs collected from the panchromatic channel, and adjusted using the relationship determined in the previous section to align to the multispectral bands. The RMS error of the 40 GCPs collected for the panchromatic image is $5.18 \mathrm{~m}$. The GCPs will have the same RMS in the multispectral domain, plus any uncertainties in the adjustment factors.

\section{Conclusions}

Good geometric correction of EO-1data is essential when comparison with other sensors is required. GCPs collected by fitting vector objects over the imagery add flexibility in choosing a GCP source. Lake boundaries served as an excellent source of GCP collection for the GVWD ETM+, Hyperion and ALI imagery. RMS errors of collected GCPs when using a Rational Function Model were at or under the $10 \mathrm{~m}$ accuracy of the vectors. Using a Rational Function Model and a digital terrain model, we geocoded the Hyperion to an RMS of $10.01 \mathrm{~m}$ and the ALI to an RMS of $5.18 \mathrm{~m}$.

Within sensor distortions require an adjustment factor be calculated and applied to GCPs that are applied to each array in Hyperion or each band in ALI. For the Hyperion sensors the correction applied to adjust GCPs collected from the VNIR to apply to the SWIR was estimated by collecting GCPs independently in both arrays. SWIR pixel coordinates can be calculated by adding 0.635 to the VNIR pixel coordinates. The SWIR's line coordinates gradually decrease across the array, the west side lines up and the east side drops down one line. Equations (2) and (3) describe the adjustment to make to the pixel-line coordinates of GCPs collected from the VNIR.

Geometric correction of the ALI can be accomplished by collecting GCPs from the panchromatic band and creating GCPs for each multi-spectral channel. The panchromatic GCP can be normalized for offset and pixel size differences. The M4 array was measured for offset. Then a separate constant was calculated to adjust the GCP pixel-line coordinates for each band. Table 2 has the measured differences of the normalized panchromatic pixel-line coordinates to each band's pixel line coordinate. There is a trend that as the band number increases, the imagery shifts down about third of a line and to the right a quarter of a pixel.

Geocorrecting hyperspectral data will affect the original spectra of ground features. A simplified comparison of three spectra extracted from the uncorrected and geocorrected imagery shows that the greatest raw DN difference was only $3.65 \%$. Future study of the effect of geo-corrected spectra on endmember extraction is required to better explain this effect.

\section{AcKNowledgments}

We acknowledge the cooperation of the Greater Victoria Watershed District and Weyerhaeuser. We are most grateful for financial support from Natural Resources Canada and the Canadian Space Agency (CSA). One of us (DGG) acknowledges support from the Natural Sciences and Engineering Research Council of Canada.

\section{REFERENCES}

[1] D. G. Goodenough, A. S. Bhogal, A. Dyk, O. Niemann, T. Han, H. Chen, C. West, and C. Schmidt, "Calibration of Forest Chemistry for Hyperspectral Analysis," Proc. IGARSS 2001, Sydney, Australia, 2001, vol. 1, pp. 52-56.

[2] J. Pearlman, "Hyperion processing evolution," personal communication to the EO-1 Science and Validation Team., 2001.

[3] J. A. Mendenhall and D. P. Ryan-Howard, "Earth Observing-1 Advanced Land Imager: Spectral Response Calibration," MIT Lincoln Laboratory August 22, 2000.

[4] C. V. Tao and Y. Hu, "A comprehensive study of the rational function model for photogrammetric processing," Photogrammetric Engineering \& Remote Sensing, vol. 67, pp. 1347-1357, 2001.

[5] T. R. McVicar, T. G. V. Niel, and D. L. B. Jupp, "Geometric Validation of Hyperion Data acquired by Earth Observing 1 Satellite at Coleambally Irrigation Area," CSIRO Land and Water, Draft XX/01, October, 2001. 